\title{
Ocorrência de parasitos gastrintestinais em cães e estudo de fatores de risco em propriedades rurais da região Oeste do Paraná, Brasil
}

\author{
[Occurrence of gastrointestinal parasites in dogs and study of risk factors \\ in rural properties in the western region of Paraná, Brazil]
}

\section{"Artigo Científico/Scientific Article"}

\author{
Alessandra Snak ${ }^{1 *}$, Arielle Aparecida Lara ${ }^{2}$, Nelson Luis Mello Fernandes ${ }^{2}$, \\ Silvia Cristina Osaki ${ }^{2}$
}

\begin{abstract}
${ }^{1}$ Departamento de Medicina Veterinária, Universidade do Estado de Santa Catarina, Lages-SC, Brasil. ${ }^{2}$ Departamento de Medicina Veterinária, Universidade Federal do Paraná, Palotina-PR, Brasil.

*Autor para correspondência/Corresponding author: E-mail: alessandra.snak@ hotmail.com
\end{abstract}

\section{Resumo}

Os cães são susceptíveis a diversas espécies de endoparasitos que, além de interferem no desenvolvimento do animal, podem propiciar o aparecimento de infecções secundárias. Parte desses parasitos são zoonoses, como Ancylostoma spp., Toxocara spp. e Giardia spp. O objetivo desse trabalho foi avaliar a ocorrência de parasitos gastrintestinais em cães pertencentes as propriedades rurais e correlacionar os principais fatores de risco associados a presença dos parasitos. Foram colhidas 120 amostras de fezes de cães de 46 propriedades rurais localizadas na região Oeste do Paraná, Brasil. Foram realizados três métodos para a pesquisa de ovos, oocistos, cistos e/ou larvas de endoparasitos, o método de Sheather, de Willis-Mollay e de Hoffman, Pons e Janer. Ainda foram coletados dados em um questionário epidemiológico para detectar os principais fatores de risco associados. Das amostras analisadas 71,67\% (86/120) foram positivas para endoparasitos, sendo os mais encontrados: Ancylostoma spp. (80,23\%), Trichuris vulpis (20,93\%), Cystoisospora spp. (17,44\%), Giardia spp. (8,14\%). Além desses parasitos também foram encontrados Sarcocystis spp., Toxocara spp., Hymenolepis diminuta e Capillaria spp. em um percentual menor. Pseudoparasitos como oocistos da família Adeleidae e Eimeria spp., e ovos da família Ascaridiidae também foram encontrados nas amostras de fezes. 29,07\% dos animais apresentaram infecção mista. Quando analisados os parasitos individualmente foi possível detectar os principais fatores relacionados com as endoparasitoses, como consistência das fezes, uso de antiparasitários e histórico de verminose.

Palavras-chave: endoparasitos; epidemiologia; zoonoses.

\begin{abstract}
Dogs are susceptible to several species of endoparasites which, in addition to interfering with the development of the animal, may lead to the appearance of secondary infections. Part of these parasites cause zoonosis, such as Ancylostoma spp., Toxocara spp., and Giardia spp. The objective of this study was to evaluate the occurrence of gastrointestinal parasites in dogs belonging to rural properties and to correlate the main risk factors associated with the presence of parasites. A total of 120 fecal samples were collected from 46 rural farms located in the Western region of Paraná, Brazil. Three methods were used to search for eggs, oocysts, cysts, and/or endoparasite larvae, the Sheather, the Willis-Mollay and the Hoffman, Pons and Janer methods. Data were also collected in an epidemiological questionnaire to detect the main associated risk factors. Of the samples analyzed, $71.67 \%$ (86/120) were positive for endoparasites, the most commonly found being: Ancylostoma spp. (80.23\%), Trichuris vulpis (20.93\%), Cystoisospora spp. (17.44\%), Giardia spp. (8.14\%). In addition to these parasites, Sarcocystis spp., Toxocara spp., Hymenolepis diminuta, and Capillaria spp. in a lower percentage were also found. Pseudoparasites, such as oocysts of the family Adeleidae and Eimeria spp., and eggs of the Ascaridiidae family were also found in fecal samples. A total of $29.07 \%$ of the animals
\end{abstract}


had mixed infection. When analyzing the individual parasites, it was possible to detect the main factors related to endoparasites such as consistency of feces, use of antiparasitics and history of verminosis.

Keywords: endoparasites; epidemiology; zoonoses.

\section{Introdução}

Os endoparasitos são encontrados em diversas espécies de animais, inclusive o homem, sendo responsáveis por várias doenças de suma importância na atualidade e estão distribuídas por todo mundo (Curi et al., 2016). Os sinais clínicos variam de acordo com o parasito e com o estado geral do hospedeiro, sendo que hospedeiros imunocomprometidos apresentam sinais mais graves do que os hospedeiros saudáveis (Leal et al., 2015).

Os cães são susceptíveis a várias espécies de endoparasitos. Esses podem interferir principalmente no desenvolvimento do animal. Geralmente a maioria dos cães são assintomáticos, porém podem apresentar sinais clínicos quando há uma grande carga parasitária ou quando o animal está imunossuprimido. Os endoparasitos podem ainda, predispor a várias infecções secundárias, prejudicando ainda mais a saúde do animal (Leal et al., 2015).

Os principais parasitos gastrintestinais encontrados em cães são os Ancylostoma spp., Trichuris spp., Toxocara spp., Cystoisospora spp., Giardia spp. e Dipylidium caninum. Muitos desses possuem caráter zoonótico, sendo o Ancylostoma spp. responsável por causar a doença popularmente conhecida como "larva migrans cutânea" ou "bicho geográfico". Já o Toxocara spp. causa a doença em humanos conhecida como "larva migrans visceral". Giardia spp. atinge principalmente crianças, idosos e imunocomprometidos, sendo uma das principais causas de surtos de diarreia em crianças que frequentam creches (Mccarthy e Moore, 2000; Labruna et al., 2006; Curi et al., 2016).

As principais vias de transmissão por parasitos gastrintestinais são pela ingestão de água ou alimentos contaminados, porém o homem ainda pode se infectar pelo contato direto com o parasito, onde larvas penetram pela pele, podendo migrar para diversos órgãos ou formar túneis subcutâneos, como no caso da larva migrans cutânea (Carvalho e Rocha, 2014; Tellería et al., 2015; Soares e Tasca, 2016).

Em propriedades rurais há um contato muito próximo de diversas espécies animais, incluindo as silvestres, podendo, os parasitos se disseminarem rapidamente, infectando um grande número de animais e o homem (Correa e Passos, 2001). Os cães, que na maioria das vezes vivem soltos, podem ser os principais responsáveis por carregar e disseminar esses parasitos para o pasto e na água, essa podendo servir para consumo humano (Robertson et al., 2000; Labruna et al., 2006).

O objetivo desse trabalho foi pesquisar a ocorrência de parasitos gastrintestinais em cães de propriedades rurais do Oeste do Paraná e correlacionar os resultados com um questionário epidemiológico, para avaliar os principais fatores de risco associados à parasitose.

\section{Material e Métodos}

No total, foram colhidas 120 amostras de fezes de cães de 46 propriedades rurais da região Oeste do Paraná, distribuídas nas cidades de Cascavel, Toledo, Marechal Cândido Rondon e Palotina (Tabela 1).

Tabela 1. Distribuição do número de propriedades e número de animais em que foram coletadas fezes em cada cidade da região Oeste do Paraná, Brasil.

\begin{tabular}{lcc}
\hline & $\mathrm{N}^{\mathbf{o}}$ de propriedades & $\mathrm{N}^{\circ}$ de animais \\
\hline Cascavel & 17 & 59 \\
Marechal & 9 & 17 \\
Cândido & 5 & 9 \\
Rondon & 15 & 35 \\
Palotina & & \\
Toledo & & \\
Total & 46 & 120 \\
\hline
\end{tabular}

As amostras de fezes colhidas foram armazenadas em recipientes com tampa de rosca e refrigeradas até análise, que ocorreu em até sete dias após a colheita.

Foram anotados os dados referentes ao sexo, raça e idade dos animais durante a colheita das amostras. Ainda, para coleta de dados foi aplicado um questionário epidemiológico que continha informações como histórico da presença de endoparasitos nas fezes, frequência do uso de antiparasitários, ambiente onde os cães residem, se os animais já apresentaram alguma doença, entre outros. 
As amostras de fezes foram submetidas a três técnicas para o encontro de ovos, oocistos, cistos e larvas de parasitos gastrintestinais: a técnica de Willis-Mollay modificada, a de Hoffman, Pons e Janer modificada e o método de Sheather modificado.

A análise estatística e a correlação dos dados do questionário epidemiológico com os resultados das análises das fezes dos cães foi realizada no programa EpiInfo (versão7.2.0.1), através do quiquadrado tabelado na ordem $2 \times 2$ e correção de Yates ( $\rho=$ ou $\geq 0,05)$.

\section{Resultados e Discussão}

A ocorrência de enteroparasitos em cães da região Oeste do Estado do Paraná foi de 71,67\% (86/120). Quando analisadas as propriedades, 71,15\% (33/46) possuíam pelo menos um cão eliminando ovos, oocistos ou cistos de parasitos nas fezes (Tabela 2). A cidade de Palotina foi a que apresentou uma maior ocorrência de cães parasitados $(77,78 \%, 7 / 9)$, já a cidade de Toledo foi a que teve um maior número de propriedades com cães positivos $(86,66 \%, 13 / 15)$.

Tabela 2. Ocorrência de enteroparasitos em cães de propriedades rurais da região Oeste do Paraná, Brasil.

\begin{tabular}{lcccccc}
\hline \multirow{2}{*}{ Cidade } & \multicolumn{3}{c}{ Animais } & \multicolumn{3}{c}{ Propriedades } \\
\cline { 2 - 7 } & Positivos & Negativos & $\%$ & Positivas & Negativas & $\%$ \\
\hline Cascavel & 43 & 16 & 72,88 & 13 & 4 & 76,47 \\
Toledo & 25 & 10 & 71,42 & 13 & 2 & 86,66 \\
Marechal Cândido Rondon & 11 & 6 & 64,7 & 7 & 2 & 77,78 \\
Palotina & 7 & 2 & 77,78 & 4 & 1 & 80 \\
Total & 86 & 34 & 71,66 & 37 & 9 & 71,15 \\
\hline
\end{tabular}

O percentual de positividade encontrado nesse trabalho foi alto quando comparado com outros autores, como Frizzo et al. (2016) que encontraram $56 \%$ de positividade quando analisaram 50 amostras de fezes de cães da área rural de Santa Catarina. Dubná et al. (2007) ao analisarem amostras de 540 cães da área rural em Praga, República Checa, e encontraram $41,7 \%$ das amostras positivas, e Curi et al. (2016) após realizarem exames coproparasitológicos de 129 cães da área rural de Minas Gerais e encontraram $58 \%$ das amostras positivas.

Alguns autores relataram um maior número de cães parasitados na área rural quando comparados com cães da área urbana (Dubná et al., 2007). Porém, outros trabalhos encontram um grande número de cães domiciliados parasitados, como Ribeiro et al. (2015) no Estado do Paraná que encontraram 60,1\% (74/123) dos cães da área urbana positivos para pelo menos um endoparasito e Labruna et al. (2006) no Estado de Rondônia que encontraram $84 \%$ das amostras positivas de cães da área urbana, positividades maiores do que as encontradas na área rural por diversos autores (Dubná et al., 2007; Curi et al., 2016; Frizzo et al., 2016). Cães errantes presentes na área urbana também apresentam alta positividade para parasitos, como verificado por Pereira Junior e Barbosa (2013) quando avaliaram fezes de 80 cães errantes no Município de Manaus e todos foram positivos para parasitos gastrintestinais.
Os endoparasitos encontrados foram ovos de Ancylostoma spp., Trichuris vulpis, Hymenolepis diminuta, Toxocara spp. e Capillaria spp., oocistos de Cystoisospora spp. e Sarcocystis spp. e cistos de Giardia spp. (Figura 1).

Ancylostoma spp., Toxocara spp. e Giardia spp. são os principais enteroparasitos com potencial zoonótico e possuem grande importância na atualidade (Babá et al., 2013; Ribeiro et al., 2015). Em propriedades rurais, cães possuem acesso a uma área ampla, podendo defecar próximo das verduras, frutas e água destinada ao consumo humano, gerando infecção e favorecendo a disseminação dessas zoonoses. Portanto, medidas preventivas devem ser adotadas para evitar a transmissão para os seres humanos.

Espécies do gênero Ancylostoma podem causar em humanos a doença conhecida como "larva migrans cutânea", quando larvas do terceiro estágio (L3) penetram através da pele e migram na epiderme, formando cordões eritematosos e pruriginosos (Tellería et al., 2015). No Brasil há diversos relatos dessa infecção, principalmente no verão, quando aumenta o número de casos (Heukelback et al., 2006; Reichert et al., 2016).

A infecção por Toxocara spp. acontece quando o ser humano ingere os ovos embrionados junto com alimentos ou com a água, no intestino os ovos eclodem, e as larvas penetram através da parede atingindo a corrente circulatória e migram para vários órgãos podendo causar diversos sinais clínicos (Carvalho e Rocha, 2014). Há vários 
trabalhos que relataram a prevalência dessa infecção em humanos, sendo considerada alta em alguns estudos (Schoenardie et al., 2013; Cassenote et al., 2014).

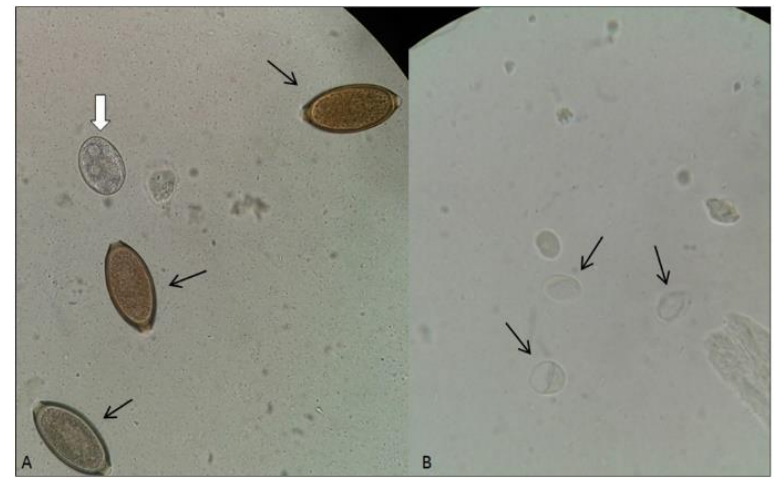

Figura 1. Ovos e cistos de enteroparasitos encontrados em fezes de cães de propriedades rurais da região Oeste do Paraná, Brasil; A) seta clara: ovo de Ancylostoma spp., seta escura: ovos de Trichuris vulpis (aumento de 400X); B) cistos de Giardia spp. (aumento de 1000X).

Já a giardíase, causada pelo protozoário Giardia spp., é transmitida principalmente através da ingestão de água e alimentos contaminados. $\mathrm{O}$ principal sinal clínico é a diarreia intermitente, acometendo principalmente crianças que frequentam creches, idosos e imunossuprimidos (Cama e Mathison, 2015; Soares e Tasca, 2016).

Além dos ovos, oocistos e cistos dos enteroparasitos, também foram encontrados ovos e oocistos de pseudoparasitos como oocistos da família Adeleidae e Eimeria spp. e ovos da família Ascaridiidae, esses são considerados pseudoparasitos, pois não parasitam os animais, apenas foram ingeridos com alimentos contaminados e eliminados através das fezes (Figura 2).

Adeleidae são parasitos de invertebrados e a presença de oocistos desse parasito em fezes de cães indicam que esses possuem o hábito de ingerir insetos. Ovos da família Ascaridiidae e oocistos de Eimeria spp. podem sugerir o hábito de coprofagia desses animais, pois todos os oocistos de Eimeria spp. já estavam esporulados. Nijse et al. (2014) em seu estudo demonstram que a coprofagia é um comportamento generalizado entre os cães, e esse hábito pode levar a resultados errôneos nas análises coproparasitológicas. Já Gressler et al. (2009) ressaltam a importância do diagnóstico correto de coccídeos e realização da prova de esporulação em placa para diferenciar protozoários da família Adeleidae e Eimeriidae e assim evitar resultados falsos.

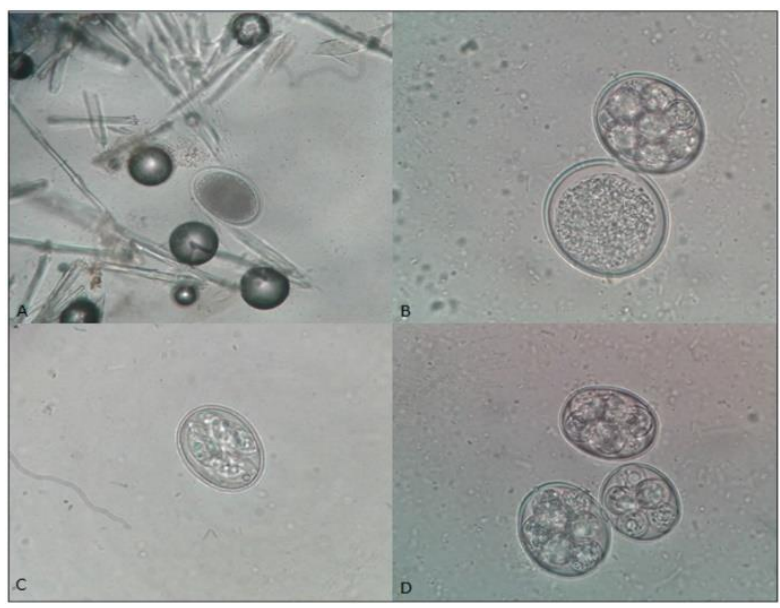

Figura 2. Pseudoparasitos encontrados em fezes de cães de propriedades rurais da região Oeste do Paraná. A) ovo de parasito da família Ascaridiidae (aumento 400X); B) oocisto esporulado e não esporulado de Adeleidae (aumento 1000X); C) oocisto esporulado de Eimeria spp. (aumento de 1000X); D) oocistos esporulados de Adeleidae (aumento de 1000X).

Os parasitos encontrados com maior frequência foram Ancylostoma spp., Trichuris vulpis, Cystoisospora spp. e Giardia spp. O total de $29 \%(25 / 86)$ das amostras positivas apresentavam mais de uma espécie de parasito, ou seja, o animal possuía infecção mista. Capillaria spp., $H$. diminuta e Sarcocystis spp. foram encontrados em apenas uma amostra (Tabela 3).

Ancylostoma spp. foi o parasito com maior ocorrência nesse trabalho, sendo o parasito mais frequente em cães, e o mais encontrado em diversos trabalhos (Ribeiro et al., 2015; Frizzo et al., 2016; Curi et al., 2016). Nos trabalhos de Labruna et al. (2006), Frizzo et al. (2016) e Curi et al. (2016), Toxocara spp. foi um dos parasitos mais frequentemente encontrado, porém nesse trabalho, assim como no trabalho de Ribeiro et al. (2015), Trichuris vulpis e o Cystoisospora spp. apresentaram um maior percentual de ocorrência, após o Ancylostoma spp.

Os percentuais encontrados de Capillaria spp. geralmente são baixos, estando associados com o ciclo do parasito (Paoletti et al., 2015; Cury et al., 2016). Hymemolepis diminuta, parasito principalmente de roedores, raramente é encontrado em cães, por esse motivo os percentuais são geralmente baixos (Doyle et al., 2006; Nqui et al., 2014; Rojekittikhun et al., 2014).

Giardia spp. foi encontrado em um percentual menor do que em outros trabalhos (Labruna et al., 2006; Frizzo et al., 2016). A baixa frequência de cistos de Giardia spp. encontrados em fezes de cães deve-se ao fato desse parasito ser 
eliminado intermitentemente, portanto para o correto diagnóstico é recomendada a coleta de mais de uma amostra de fezes, em um período de no mínimo três dias. Bouzid et al. (2015) sugeriram a utilização de outros métodos para o diagnóstico da parasitose devido à baixa eficiência do método de microscopia.

Tabela 3. Frequências dos enteroparasitos encontrados em fezes de cães de propriedades rurais da região Oeste do Paraná, Brasil.

\begin{tabular}{lcccccc}
\hline & \multicolumn{3}{c}{ Total amostras $(\mathrm{n}=120)$} & \multicolumn{2}{c}{ Total amostras positivas $(\mathrm{n}=86)$} \\
\cline { 2 - 7 } & Positivo & Negativo & $\%$ & Positivo & Negativo & $\%$ \\
\hline Ancylostoma spp. & 69 & 51 & 57,5 & 69 & 17 & 80,23 \\
Capillaria spp. & 1 & 119 & 0,83 & 1 & 85 & 1,16 \\
Cystoisospora spp. & 15 & 105 & 12,5 & 15 & 71 & 17,44 \\
Giardia spp. & 7 & 113 & 5,83 & 7 & 79 & 8,14 \\
H. diminuta & 1 & 119 & 0,83 & 1 & 85 & 1,16 \\
Sarcocystis spp. & 1 & 119 & 0,83 & 1 & 85 & 1,16 \\
Toxocara spp. & 4 & 116 & 3,33 & 4 & 82 & 4,65 \\
Trichuris vulpis & 18 & 102 & 15 & 18 & 68 & 20,93 \\
Pseudoparasito & 6 & 114 & 5 & 6 & 80 & 6,97 \\
Infecção mista & 25 & 95 & 20,83 & 25 & 61 & 29,06 \\
\hline
\end{tabular}

Sarcocystis spp. foi encontrado em apenas uma amostra, representando $1,16 \%(1 / 86)$ das amostras positivas. Ribeiro et al. (2015) encontraram esporocistos desse protozoário em quatro animais, já Labruna et al. (2006) encontraram em $18,9 \%$ das amostras pesquisadas, percentuais maiores do que o encontrado nesse trabalho. Isso pode ter sido influenciado pela alimentação fornecida para os cães, favorecendo ou dificultando a infecção, pois esse parasito é transmitido pelo consumo de carne crua ou mal passada.

Das amostras colhidas, 107 eram de cães adultos, sendo que $70,10 \%$ foram positivas para algum endoparasito. Quando analisadas as fezes dos filhotes, cerca de $84,61 \%$ (11/13) foram positivas. Apesar dos filhotes terem apresentando uma frequência maior de enteroparasitos, a idade não foi identificada como fator de risco nesse trabalho, pois não houve diferença significativa $(p=0,440)$. Ribeiro et al. (2015) encontraram a idade como fator de risco para infecções de Ancylostoma spp. e Trichuris vulpis, com cães adultos apresentaram maior chance de ter o parasito. Já Curi et al. (2016) encontram uma maior chance de filhotes ter Toxocara spp. do que adultos.

A maioria das amostras de fezes era de cães machos e dessas $68,35 \%$ (54/79) foram positivas. As fêmeas, apesar de um número menor de amostras, apresentaram uma maior positividade, cerca de 78,04\% (32/41). Ribeiro et al. (2015) também não encontraram diferença significativa entre os sexos dos animais. Já Curi et al. (2016) encontraram cães machos como fator de risco para infecções mistas.

A raça dos animais também não apresentou diferença significativa, 74,17\% (89/120) dos cães que foram colhidas amostras de fezes não apresentavam raça definida, e desses $76,40 \%$ eram positivos. Dos cães com raça definida o percentual de positividade foi menor, cerca de 58\% (18/31). Esses resultados, apesar de serem apenas diferenças numéricas, podem demonstrar que tutores apresentam um maior cuidado sanitário com cães de raça quando comparado com cães sem raça definida. Resultados semelhantes foram encontrados por Ribeiro et al. (2015) quando analisaram 123 amostras de cães domiciliados e encontraram um maior número de cães sem raça definida parasitados.

Durante a realização dos exames era anotada a consistência das fezes dos animais. A maioria apresentou aspecto normal. Seis apresentavam-se de pastosa a líquida e, dessas, cinco foram positivas e todas apresentavam infecção por protozoário. Quatro possuíam oocistos de Cystoisospora spp., uma continha cistos de Giardia spp., uma possuía oocistos de Sarcocystis spp. e duas amostras apresentavam ovos de helmintos, Trichuris vulpis e Ancylostoma spp.

A consistência das fezes pode estar associada com doenças parasitárias, principalmente as causadas por protozoário, pois o principal sinal clínico provocado por protozoários entéricos é a diarreia, geralmente por má absorção (Pércope, 2015). Fezes pastosas obteve associação com a infecção por alguns protozoários como 
Cystoisospora spp. (p=0,0004), ou seja, um cão com fezes pastosas possuía 18,72 vezes mais chance de ter esse protozoário do que um cão com fezes normal. Sarcocystis spp. é outro protozoário que também é encontrado com mais chance em fezes pastosas $(\mathrm{p}=0,03)$.
Quando avaliada a positividade no geral não houve fatores de risco envolvidos. Porém, quando analisados os fatores de risco por parasito, alguns foram considerados significativos (Tabela 4), tais como: consistência de fezes, histórico de parasitos e uso de antiparasitários.

Tabela 4. Fatores de risco associados com enteroparasitos encontrados em amostras de fezes de cães de propriedades rurais da região Oeste do Estado do Paraná, Brasil.

\begin{tabular}{|c|c|c|c|c|c|c|c|c|c|c|c|c|c|}
\hline \multirow{2}{*}{\multicolumn{2}{|c|}{ Variáveis }} & \multicolumn{2}{|c|}{ Ancylostoma spp. } & \multicolumn{2}{|c|}{ Cystoisospora spp. } & \multicolumn{2}{|c|}{ Giardia spp. } & \multicolumn{2}{|c|}{ Sarcocystis spp. } & \multicolumn{2}{|c|}{ Trichuris vulpis } & \multicolumn{2}{|c|}{ Infecção mista } \\
\hline & & $\mathbf{O R} * * *$ & p***** & OR & p & OR & $\mathbf{p}$ & OR & $\mathbf{p}$ & OR & $\mathbf{p}$ & OR & $\mathbf{p}$ \\
\hline Condição & $\begin{array}{l}\text { preso } \\
\text { solto } \\
\text { preso e solto }\end{array}$ & $\begin{array}{l}3,54(1,1-11,3) \\
\text { ns* } \\
\text { ns }\end{array}$ & $\begin{array}{l}0,047 \\
1 \\
0,12\end{array}$ & $\begin{array}{l}\text { ns } \\
4,00(1,26-12,60) \\
\text { ns }\end{array}$ & $\begin{array}{l}0,138 \\
0,027 \\
0,446\end{array}$ & $\begin{array}{l}\text { ns } \\
11,38(1,32-97,9) \\
\text { ns }\end{array}$ & $\begin{array}{l}0,485 \\
0,02 \\
0,182\end{array}$ & $\begin{array}{l}\mathrm{ns} \\
\mathrm{ns} \\
\mathrm{ns}\end{array}$ & $\begin{array}{l}1 \\
1 \\
0,933\end{array}$ & $\begin{array}{l}\mathrm{ns} \\
\mathrm{ns} \\
3,71(1,23- \\
11,2)\end{array}$ & $\begin{array}{l}0,731 \\
0,08 \\
0,029\end{array}$ & $\begin{array}{l}\mathrm{ns} \\
\mathrm{ns} \\
\mathrm{ns}\end{array}$ & $\begin{array}{l}1 \\
0,953 \\
1\end{array}$ \\
\hline Alimentação & $\begin{array}{l}\text { Comida caseira } \\
\text { Ração } \\
\text { Comida e ração }\end{array}$ & $\begin{array}{l}\text { ns } \\
\text { ns } \\
\text { ns }\end{array}$ & $\begin{array}{l}0,625 \\
0,608 \\
0,327\end{array}$ & $\begin{array}{l}\mathrm{ns} \\
\mathrm{ns} \\
\mathrm{ns}\end{array}$ & $\begin{array}{l}0,17 \\
0,26 \\
1\end{array}$ & $\begin{array}{l}\text { ns } \\
\text { ns } \\
\text { ns }\end{array}$ & $\begin{array}{l}0,658 \\
0,968 \\
1\end{array}$ & $\begin{array}{l}\mathrm{ns} \\
\mathrm{ns} \\
\mathrm{ns}\end{array}$ & $\begin{array}{l}1 \\
1 \\
1\end{array}$ & $\begin{array}{l}\text { ns } \\
\text { ns } \\
\text { ns }\end{array}$ & $\begin{array}{l}0,562 \\
0,156 \\
0,005 \\
7\end{array}$ & $\begin{array}{l}\text { ns } \\
\text { ns } \\
\text { ns }\end{array}$ & $\begin{array}{l}1 \\
0,277 \\
0,382\end{array}$ \\
\hline $\begin{array}{l}\text { Histórico de } \\
\text { verminose }\end{array}$ & & Ns & 1 & ns & 0,12 & ns & 0,851 & ns & 0,707 & $\begin{array}{l}5,55(1,89- \\
16,2)\end{array}$ & 0,002 & $3,56(1,43-8,87)$ & 0,009 \\
\hline $\begin{array}{c}\text { Uso de } \\
\text { vermíifugo }\end{array}$ & $\begin{array}{l}\text { não utiliza } \\
3 \text { meses } \\
6 \text { meses } \\
1 \text { ano } \\
>1 \text { ano }\end{array}$ & $\begin{array}{l}\text { ns } \\
\text { ns } \\
\text { ns } \\
\text { ns } \\
\text { ns }\end{array}$ & $\begin{array}{l}0,192 \\
0,37 \\
0,548 \\
0,285 \\
0,65\end{array}$ & $\begin{array}{l}\mathrm{ns} \\
\mathrm{ns} \\
\mathrm{ns} \\
4,86(1,5-15,3) \\
\mathrm{ns}\end{array}$ & $\begin{array}{l}0,108 \\
0,563 \\
0,084 \\
0,01 \\
0,215\end{array}$ & $\begin{array}{l}\text { ns } \\
\text { ns } \\
\text { ns } \\
6,59(1,36-31,9) \\
\text { ns }\end{array}$ & $\begin{array}{l}0,676 \\
0,316 \\
1 \\
0,03 \\
0,316\end{array}$ & $\begin{array}{l}\mathrm{ns} \\
\mathrm{ns} \\
\mathrm{ns} \\
\mathrm{ns} \\
\mathrm{ns}\end{array}$ & $\begin{array}{l}1 \\
0,508 \\
1 \\
1 \\
1\end{array}$ & $\begin{array}{l}\text { ns } \\
\text { ns } \\
\text { ns } \\
\text { ns } \\
\text { ns }\end{array}$ & $\begin{array}{l}0,344 \\
0,133 \\
0,224 \\
0,537 \\
0,342\end{array}$ & $\begin{array}{l}\mathrm{ns} \\
\mathrm{ns} \\
\mathrm{ns} \\
\mathrm{ns} \\
\mathrm{ns}\end{array}$ & $\begin{array}{l}0,342 \\
1 \\
0,778 \\
0,329 \\
0,09\end{array}$ \\
\hline $\begin{array}{l}\text { Consistência } \\
\text { das fezes }\end{array}$ & $\begin{array}{l}\text { Normal } \\
\text { Pastosa }\end{array}$ & $\begin{array}{l}\text { ns } \\
\text { ns }\end{array}$ & $\begin{array}{l}0,42 \\
0,42\end{array}$ & $\begin{array}{l}0,05(0,008-0,32) \\
18,72(3,07-114,1)\end{array}$ & $\begin{array}{l}0,0004 \\
0,0004\end{array}$ & $\begin{array}{l}\mathrm{ns} \\
\mathrm{ns}\end{array}$ & $\begin{array}{l}0,788 \\
0,788\end{array}$ & $\begin{array}{l}\text { in* } \\
* \\
\text { in* } \\
*\end{array}$ & $\begin{array}{l}0,03 \\
0,03\end{array}$ & $\begin{array}{l}\mathrm{ns} \\
\mathrm{ns}\end{array}$ & $\begin{array}{l}0,481 \\
0,481\end{array}$ & $\begin{array}{l}\text { ns } \\
\text { ns }\end{array}$ & $\begin{array}{l}0,197 \\
0,197\end{array}$ \\
\hline $\begin{array}{l}\text { Histórico de } \\
\text { doença }\end{array}$ & & Ns & 0,326 & $\mathrm{~ns}$ & 0,81 & ns & 0,244 & ns & 0,579 & $\begin{array}{l}6,44(2,21- \\
18,7)\end{array}$ & $\begin{array}{l}0,000 \\
6\end{array}$ & ns & 0,592 \\
\hline
\end{tabular}

Nesse estudo infecções por Toxocara spp., Capillaria spp. e Hymenolepis diminuta não apresentaram fatores de risco associados, um dos motivos pode ser o número baixo de amostras positivas para esses parasitos.

Já o parasitismo por Ancylostoma spp. apresentou como principal fator de risco o fato do cão viver preso, com um OR (odds ratio) de 3,54. $\mathrm{O}$ fato do cão sempre estar no mesmo ambiente facilita a autoinfecção, devido o ambiente contaminado, justificando a associação de Ancylostoma spp. com essa condição, como já relatado por Pinto et al. (2007).

Os cães que vivem soltos possuíram quatro e 11,38 vezes mais chance de estarem parasitados por Cystoisospora spp. e Giardia spp., respectivamente. Já os cães que ficaram um período do dia soltos e outro período presos tiveram 3,71 vezes mais chance de um parasitismo por Trichuris vulpis. Esses resultados podem ser justificados pelo fato do animal livre ter uma maior chance de contato com outros animais ou com alimentos e água contaminadas com parasitos.

O histórico de enteroparasitos foi atribuído como um fator de risco para o Trichuris vulpis, com um OR de 5,55 e para as infecções mistas que possuem 3,56 vezes mais chance de acontecer quando há a presença desse fator.
A utilização de vermífugo uma vez por ano foi fator de risco para dois protozoários, o Cystoisospora spp. $(\mathrm{OR}=4,86)$ e a Giardia spp. $(\mathrm{OR}=6,59)$. Os vermífugos comumente utilizados por proprietários não são eficazes para o tratamento de protozoários entéricos. Acredita-se que seja esse o motivo da associação com esse fator de risco. Já histórico de doenças, como viroses, foram fatores de risco para o parasitismo por Trichuris vulpis, apresentando um OR de 6,44, podendo estar associado principalmente com o manejo sanitário, pois cães com condições de vida precárias possuem uma maior chance de estarem parasitados, como também de adquirir outras doenças (Menão et al., 2014).

\section{Conclusão}

A ocorrência de enteroparasitos em cães da região Oeste do Paraná é alta. Os principais parasitos encontrados foram Ancylostoma spp., Trichuris vulpis, Cystoisospora spp., Toxocara spp. e Giardia spp.

Pseudoparasitos como oocistos da família Adeleidae, Eimeria spp. e ovos da família Ascaridiidae foram encontrados em algumas amostras, indicando o hábito de coprofagia e entomogafia dos cães. 
Os principais fatores de risco encontrados estão relacionados com a rotina de uso de vermífugos, histórico de verminose, condição de sobrevivência dos animais (livres ou presos), histórico de doenças e presença de carrapatos.

\section{Conflito de Interesse}

Os autores declaram não existir conflito de interesse.

\section{Comitê de Ética}

Esse estudo está de acordo com os Princípios Éticos da Experimentação Animal, adotado pelo Colégio Brasileiro de Experimentação e foi aprovado pela Comissão de Ética no Uso de Animais da Universidade Federal do Paraná-Setor Palotina, com protocolo número 50/2014.

\section{Referências}

Anderson, M.R.; May, R.M. Population biology of infectious diseases: Part I. Nature, 280(2):361-367, 1979.

Babá, A.Y.; Obara, A.T.; Silva, E.S. Levantamento do Conhecimento de Proprietários de Cães Domésticos Sobre Zoonoses. UNOPAR Cientifica Ciências Humanas e Educação, 14(3): 251-258, 2013.

Bouzid, M.; Halai, K.; Jeffreys, D.; Hunter, P.H. The prevalence of Giardia infection in dogs and cats, a systematic review and metaanalysis of prevalence studies from stool samples. Veterinary Parasitology, 207(34):181-202, 2015.

Cama, V.A.; Mathison, B.A. Infections by intestinal Coccidia and Giardia duodenalis. Clinics in Laboratory Medicine, 2(1):423444, 2015.

Carvalho, E.A.A.; Rocha, R.L. Visceral larva migrans syndromes associated with toxocariasis: epidemiology, clinical and laboratory aspects of human toxocariasis. Current Tropical Medicine Reports, 1(1):74-79, 2014.

Cassenote, A.J.F.; De Abreu Lima, A.R.; Pinto Neto, J.M.; Rubinsky-Elefant, G. Seroprevalence and Modifiable Risk Factors for Toxocara spp. in Brazilian Schoolchildren. PLOS Neglected Tropical Diseases, 8(5): e2830, 2014.

Corrêa, S.H.R.; Passos, E.C. Wild animals and public health. In: Fowler, M.E.; Cubas, Z.S. Biology, medicine, and surgery of South
American wild animals. Ames: Iowa University Press, 2001. p. 493-499.

Curi, N.H.A.; Paschoal, A.M.O.; Massara, R.L.; Santos, H.A.; Guimarães, M.P.; Passamani, M.; Chiarello, A.G. Risk factors for gastrointestinal parasite infections of dogs living around protected areas of the Atlantic Forest: implications for human and wildlife health. Brazilian Journal of Biology, 77(2): 388-395, 2016.

Doyle, R.L.; Monteiro, S.G.; Graça, D.L.; Santurio, J.M.; Silva, A.S.; Bertolin, K. Helmintologic evaluation of mice (Mus musculus) raised in an experimental mouse house. Revista da FZVA, 13(2):108-115. 2006.

Dubna, S.; Langrova, I.; Na'Pravni'K, J.; Jankovska, I.; Vadlejch, J.; Peka, R.S.; Fechtner, J. The prevalence of intestinal parasites in dogs from Prague, rural areas, and shelters of the Czech Republic, Veterinary Parasitology, 145(1):120-128, 2007.

Frizzo, C.; Schimidt, A.P.; Wagner, G.; Muller, G.A. Intestinal parasites present in canine fecal samples collected in rural areas of municipalities in the midwest of Santa Catarina, Brazil. Revista de Patologia Tropical, 45(2):227-232, 2016.

Gressler, L.T.; Da Silva, A.S.; Oliveira, C.B.; Soares, J.F.; Monteiro, S.G. Occurrence of pseudoparasite coccidians in carnivores. Archives of Veterinary Science, 14(2):9195, 2009.

Heukelback, J.; Wilcke, T.; Feldmeier, H. Cutaneous larva migrans (creeping eruption) in an urban slum in Brazil. International Journal of Dermatology, 45(7):511-515, 2004.

Hoffman, W.A.; Pons, J.A.; Janer, J.L. The sedimentation concentration method in Schistosomiasis mansoni. Journal of Public Health, 9(1):281-289, 1934.

Labruna, M.B.; Pena, H.F.J.; Souza, S.L.J.; Pinter, A.; Silva, J.C.R. Ragozo, A.M.A.; Camargo, L.M.A.; Gennari, S.M. Prevalência de endoparasitas em cães da área urbana do município de Monte Negro, Rondônia. Arquivo do Instituto Biológico, 73(2):183193, 2006.

Leal, P.D.A.; Moraes, M.I.M.R.; Barbosa, L.L.O.; Figueiredo, L.P.; Silva, S.L.; Lopes, C.W.G. Parasitos gastrintestinais em cães domiciliados atendidos em serviço de saúde animal, Rio de Janeiro, Brasil. Revista 
Brasileira de Medicina Veterinária, 37(1): 37-44, 2015.

Mccarthy, J.; Moore, T.A. Emerging helminth zoonoses. International Journal for Parasitology, 30(12-13):1351-1359, 2000.

Menão, M.C.; Faria, T.L.S.A.; Almeida, J.M.S.; Chiacchio, R.G.; Rocha, A. Pesquisa de infecções parasitárias com potencial zoonótico em animais mantidos em habitações coletivas. Atas De Saúde Ambiental, 2(1):28, 2014

Ngui, R.; Lee, S.C.; Yap, N.J.; Tan, T.K.; Aidil, R.M.; Chua, K.H.; Aziz, S.; Sulaiman, W.Y.; Ahmad, A.F.; Mahmud, R.; Lian, Y.L. Gastrointestinal parasites in rural dogs and cats in Selangor and Pahang states in Peninsular Malaysia. Acta Parasitology, 59(4):737-744, 2014

Nijsse, R.; Mughini-Gras, L.; Wagenaar, J.A.; Ploeger, H.W. Coprophagy in dogs interferes in the diagnosis of parasitic infections by faecal examination. Veterinary Parasitology, 204(3-4):304-309, 2014.

Paoletti, B.; Traversa, D.; Iorio, R.; De Berardini, S.A.; Bartolini, R.; Salini, R.; Di Cesare, A. Zoonotic parasites in feces and fur of stray and private dogs from Italy. Parasitology Research, 114(6):2135-2141, 2015.

Pércope, S. Diarreia aguda. Pediatria Moderna, 51(4): 141-148, 2015.

Pereira Junior, G.; Barbosa, P.S. Prevalência de endoparasitas em cães errantes na cidade de Manaus-AM. Acta Biomedica Brasiliensia, 4(2): 52-57, 2013.

Pinto, L.D.; Marques, S.M.T.; Bigatti, L.E.; Araujo, F.A.P. Enteroparasitos de cães: prevalência e conhecimento dos proprietários sobre fatores epidemiológicos. Veterinária em Foco, 5(1):10-15, 2007.

Reichert, F.; $\quad$ Pilger, D.; $\quad$ Schuster, A.; Lesshafft, H.; Guedes De Oliveira, S.; Gnautius, R.; Feldmeier, H. Prevalence and Risk Factors of Hookworm-Related Cutaneous Larva Migrans (HrCLM) in a
Resource-Poor Community in Manaus, Brazil. PLOS Neglected Tropical Diseases, 10(3): e0004514, 2016.

Ribeiro, C.M.; Lima, D.E.; Katagiri, S. Infecções por parasitos gastrintestinais em cães domiciliados e suas implicações na transmissão zoonótica. Veterinária e Zootecnia, 22(2):238-244, 2015.

Robertson, I.D.; Irwin, P.J.; Lymbery, A.J.; Thompson, R.C.A. The role of companion animals in the emergence of parasitic zoonoses. International Journal for Parasitology, 30(12):1369-1377, 2000.

Rojekittikhun, W.; Chaisiri, K.; Mahittikorn, A.; Pubampen, S.; Sa-Nguankiat, S.; Kusolsuk, T.; Maipanich, W.; Udonsom, R.; Mori, H. Gastrointestinal parasites of dogs and cats in a refuge in Nakhon Nayok, Thailand. Southeast Asian Journal of Tropical Medicine and Public Health, 45(1):31-39, 2014.

Schoenardie, E.R.; Scaini, C.J.; Brod, C.S.; Pepe, M.S.; Villela, M.M.; Mcbride, A.J.A.; Borsuk, S.; Berne, M.E.A. Seroprevalence of Toxocara Infection in Children from Southern Brazil. Journal of Parasitology, 99(3):537539, 2013.

Sheather, A.L. The detection of intestinal protozoa and mange parasites by a floatation technique. Journal of Comparative Pathology and Therapeutics, 36(4): 266-275, 1923.

Soares, R.; Tasca, T. Giardiasis: an update review on sensitivity and specificity of methods for laboratorial diagnosis. Journal of Microbiological Methods, 129: 98-102, 2016.

Telleria, R.; Bujan, M.M.; Cervini, A.B. Resolucion del casopresentadoen el número anterior - Larva migrans cutânea. Archivos Argentinos de Pediatria, 113(4): 375-377, 2015 .

Willis, I.I. A simple levitation method for the detection of hookworm ova. Medical Journal of Australia, 2(18): 375-376, 192. 